\title{
CHANGEMENT LINGUISTIQUE ET CHANGEMENT PHONETIQUE
}

\section{LINGUISTIC CHANGE AND PHONETIC CHANGE}

\author{
Anne-Gaëlle Toutain \\ Institut de langue et de littérature françaises de l'Université de Berne, \\ Laboratoire Histoire des théories linguistiques (UMR 7597, Paris), Suisse
}

Résumé: Cet article se propose d'étudier le destin du concept néogrammairien de "loi phonétique " dans l'œuvre de Saussure, Hjelmslev, Jakobson et Martinet. Nous nous efforçons tout d'abord de montrer comment Saussure réélabore cette notion dans le cadre d'une définition radicalement nouvelle de la langue. Nous nous attachons ensuite à la mise au jour des enjeux du refus structuraliste de la notion de changement phonétique aveugle, qui témoigne d'une mécompréhension significative de la conception saussurienne du changement linguistique. Cette mécompréhension conduit à une double contradiction : une assomption, en dépit d'un rejet affirmé de celle-ci, de la représentation néogrammairienne de la langue, et une représentation contradictoire de la langue et du changement linguistique.

Mots-clés: Valeur; Structuralisme; Rupture Epistémologique; Synchronie/Diachronie; Loi phonétique.

Abstract: The aim of this paper is to study the destiny of the Neogrammarian concept of "phonetic law " in the work of Saussure, Hjelmslev, Jakobson and Martinet. We first show the manner Saussure elaborates this notion, in the frame of a radically new definition of language. We then demonstrate that the structuralist refusal of the notion of blind phonetic changes manifests a significative misunderstanding of the Saussurean conception of linguistic changes, leading to a twofold contradiction: an acceptance of the Neogrammarian representation of language, while rejected, and an unconsistent representation of language and linguistic change. Keywords: Value; Structuralism; Epistemological Rupture; Synchrony/Diachrony; Phonetic law.

Les débats relatifs au concept néogrammairien de "loi phonétique " sont loin d'être clos aujourd'hui, l'explication du changement linguistique demeurant toujours pendante, en dépit de la multiplication et de la diversification des apports théoriques. Nous nous proposons ici d'étudier le destin de ce concept dans les constructions théoriques de Saussure, Hjelmslev, Jakobson et Martinet. Comme on le sait, la théorisation saussurienne du changement linguistique se fonde sur la distinction néogrammairienne entre «lois phonétiques », physiologiquement conditionnées et à effet perturbateur, 
et analogie, psychologiquement conditionnée et à effet régularisateur. La notion de "loi phonétique " est conservée par les structuralistes, mais également considérablement amendée par eux. Ces derniers, en particulier, et notamment Jakobson et Martinet, s'inscrivent en faux contre la notion de changements phonétiques " aveugles ", à laquelle ils substituent une conception structurale et téléologique du changement, linguistique en général, et phonologique en particulier. Ils affirment ainsi rompre avec la représentation " atomiste " qu'ils attribuent aux Néogrammairiens, que Saussure leur paraît avoir conservée, et, par ailleurs, poursuivre l'œuvre saussurienne en étendant à la diachronie la conception structurale que celuici aurait établie en synchronie.

Nous nous efforcerons pour notre part de montrer qu'une telle représentation témoigne d'une mécompréhension significative de la conception saussurienne du changement linguistique, significative en ce qu'en dépit des innovations théoriques du structuralisme, la représentation de la langue demeure celle des Néogrammairiens.

Nous verrons ainsi tout d'abord que Saussure, loin de se contenter de reprendre à son compte la distinction néogrammairienne entre analogie et changement phonétique, lui confere une signification nouvelle, dans le cadre de l'élaboration des concepts de système et de valeur, et d'une définition de la langue qui constitue une rupture épistémologique, au sens de Gaston Bachelard. Nous verrons ensuite comment cette rupture épistémologique est recouverte par le structuralisme européen, qui ne la perçoit pas, substituant au concept saussurien de système la notion, toute différente, de structure, et demeurant, corrélativement, tributaire de la représentation organiciste des premiers comparatistes.

\section{La conception saussurienne du changement linguistique}

La distinction entre synchronie et diachronie est une des premières à prendre forme dans la linguistique générale saussurienne, comme en témoigne la lettre à Gaston Paris du 30 décembre 1891 (DECIMO, 1994). Saussure y fait allusion à un "travail tout nouveau " (DECIMO, 1994, p. 78) où l'a conduit la préparation des conférences de novembre 1891, et qui est très probablement "De l'essence double du langage " (SAUSSURE, 2002). Dans ce manuscrit que la plus grande part des saussuriens s'accorde à dater du début des années 1890, sinon de 1891, apparaissent d'emblée, corrélativement, les deux concepts eux-mêmes inséparables de valeur et de 
système.

Le concept saussurien de valeur pose l'inséparabilité de la combinaison constitutive du signe (la combinaison entre pensée et phonie, devenant ainsi signifié et signifiant) et de la délimitation qui constitue chaque signifiant et chaque signifié comme distincts des autres signifiants ou des autres signifiés. Il définit l'entité linguistique comme une entité purement oppositive, relative, négative, dans la mesure où elle consiste en cette délimitationcombinaison constitutive du signe, et non en le signe lui-même, au sens où on l'avait défini jusque là, et qui apparaît, dans sa positivité, comme un effet de cette délimitation-combinaison. Aussi, précisément, ce concept de valeur est-il inséparable de la distinction entre synchronie et diachronie : une entité purement oppositive, relative, négative ne saurait avoir d'existence dans le temps. Saussure écrit ainsi dans «De l'essence double du langage ":

- L'échange, comme seule expression véritable de tout mouvement dans la langue.

Il y a deux sortes d'échange, qui sont complètement distincts, dans la vie de la langue, mais il n'y a point en revanche de changement. Pour qu'il y eût changement, il faudrait qu'il y eût une matière définie en elle-même à un moment donné ; c'est ce qui n'arrive jamais ; on ne prononce un mot que pour sa valeur (SAUSSURE, 2002, p. 60).

On lit de même ensuite dans la note "Sémiologie " (SAUSSURE, 2002), considérée comme contemporaine des cours de linguistique générale (1908-1912):

Par le fait qu'aucun élément n'existe (ou par mille autres raisons, car nous ne prétendons pas faire une sorte de système cartésien de choses qui tombent sous le sens de tous les côtés), on voit qu'aucun élément n'est (à plus forte raison) en état de se transformer (SAUSSURE, 2002, p. 266).

On réduit souvent la distinction saussurienne entre synchronie et diachronie à une distinction méthodologique. Il apparaît cependant clairement, à la lecture de telles propositions, que cette distinction, loin de porter sur un objet défini d'avance, qu'il s'agirait de considérer de deux points de vue distincts, rompt au contraire avec la présupposition d'un tel objet, qu'elle construit de manière radicalement nouvelle, comme double. Saussure écrit ainsi par exemple dans "Status et motus " (18941897 ?, SAUSSURE, 2002), que la linguistique lui apparaît comme " une 
science qui essaie d'assembler en un seul tout deux objets complètement disparates depuis le principe, en se persuadant qu'ils forment un seul objet " (SAUSSURE, 2002, p. 226). La distinction synchronie/diachronie opère donc une rupture avec la représentation de la langue comme une entité et, en particulier, à l'époque de Saussure, avec l'organicisme des comparatistes : au lieu d'un objet existant dans le temps, et se modifiant au fil de ce dernier, un fonctionnement (celui de la délimitation-combinaison) exclusivement synchronique, le point de vue diachronique construisant pour sa part un objet distinct et irréductible au premier. Elle est par ailleurs inséparable, là encore comme corrélat du concept de valeur, de l'opposition entre son et signe, phonologie et linguistique, qui se substitue à l'opposition traditionnellement définitoire du signe : entre son et sens. Cette substitution de l'opposition son/signe à l'opposition son/sens apparaît par exemple dans ce passage de «De l'essence double du langage » :

Le dualisme profond qui partage le langage ne réside pas dans le dualisme du son et de l'idée, du phénomène vocal et du phénomène mental ; c'est là la façon facile et pernicieuse de le concevoir. Ce dualisme réside dans la dualité du phénomène vocal COMME TEL, et du phénomène vocal COMME SIGNE - du fait physique, (objectif) et du fait physico-mental (subjectif), nullement du fait " physique " du son par opposition au fait " mental » de la signification. Il y a un premier domaine, intérieur, psychique, où existe le signe autant que la signification, l'un indissolublement lié à l'autre ; il y en a un second, extérieur, où n'existe plus que le "signe "; mais à cet instant le signe réduit à une succession d'ondes sonores ne mérite pour nous que le nom de figure vocale (SAUSSURE, 2002, p. 20-21).

Elle se double d'une redéfinition de l'opposition phonétique/ morphologie dans le cadre de la distinction diachronie/synchronie, qui est notamment lisible dans cet autre passage de "De l'essence double du langage » :

Le fait qu'il n'y a rien d'instantané qui ne soit morphologique (ou significatif); et qu'il n'y a rien non plus de morphologique qui ne soit instantané est inépuisable dans les développements qu’il comporte. Mais ce premier fait a pour contre-partie immédiate: qu'il n'y a rien de successif qui ne soit phonétique (ou hors de la signification), et qu'il n'y a rien de phonétique qui ne soit successif (SAUSSURE, 2002, p. 41). 
Or, c'est avant tout à cette redéfinition, à cette opposition entre son et signe que renvoie la distinction saussurienne entre loi phonétique et analogie, ou plus exactement, c'est dans le cadre de cette distinction entre son et signe que Saussure reprend à son compte cette distinction néogrammairienne, à laquelle il confere ainsi une signification nouvelle. La distinction est évoquée avec quelque détail dans les conférences de 1891 puis, notamment, dans le premier cours. Dans la deuxième conférence à l'université de Genève (SAUSSURE, 2002, p. 159 sqq.), l'analogie est définie, de manière traditionnelle, comme un phénomène, psychologique, par opposition au changement phonétique, qui est physiologique et physique, conscient, tandis que le changement phonétique est inconscient, et enfin, doté d'un but et d'un sens, alors que le changement phonétique est " aveugle ». Dans le premier, puis dans le deuxième cours, elle est redéfinie comme un phénomène significatif et, corrélativement, synchronique. Citons notamment:

\begin{abstract}
Tout ce qui est dans le synchronique d'une langue y compris l'analogie (= conséquence de notre activité) se résume très bien dans le terme de grammaire dans sa conception très voisine de l'ordinaire. On voit intituler un traité du jeu d'échecs grammaire, <de même la grammaire de la bourse :> implique un objet complexe et systématique, <s'applique à un système qui met en jeu des valeurs.> Il n'y a pas <pour nous> de grammaire historique, <les termes jurent ensemble :> il n'y a pas de système qui puisse être à cheval sur une suite d'époques. Ce qui est synchroniquement dans une langue est un équilibre qui se réalise de moment en moment. Par grammaire historique on veut dire linguistique diachronique, ce qui est autre chose et est condamné à n'être jamais grammatical. grammatical = significatif $=$ ressortissant à un système de signes $=$ synchronique ipso facto (SAUSSURE, 1997, p. 62).
\end{abstract}

Concernant le changement phonétique, Saussure critique à de nombreuses reprises le terme de loi - qu'il conserve cependant, faute de mieux - et cette critique est extrêmement significative. Il compare la pertinence de ce terme en matière de synchronie et de diachronie. En synchronie, ainsi qu'il le pose dans le troisième cours (SAUSSURE, 2005, p. 269), la loi n'est ni " dynamique ", ni "impérative ». En revanche, il y a bien régularité. La loi diachronique, au contraire, est dynamique et dotée d'un caractère impératif : "Il y a une force en elle. " (SAUSSURE, 2005, p. 269), lit-on de même dans le troisième cours. C'est la notion de régularité qui pose 
problème dans ce cas, et les différentes affirmations saussuriennes, à cet égard, témoignent d'une hésitation, ou d'une difficulté. Dans le deuxième cours, par exemple, Saussure affirme que " [p] our les lois phonétiques nous percevons une régularité par illusion" (SAUSSURE, 1997, p. 48). On lit en revanche dans le "Cours de phonétique 1909-1910" publié par MarieJosé Reichler-Béguelin (1980), à propos d'un hypothétique terme que l'on puisse substituer à celui, critiquable, de loi : « Il faudrait qu'il ne fit oublier ni l'un ni l'autre, que la loi phonétique a un côté accidentel, historique, et un côté régulier. " (REICHLER-BEGUELIN, 1980, p. 26).

Il nous semble que cette hésitation est révélatrice de la nature de la langue ainsi que des enjeux de la redéfinition saussurienne de l'opposition entre lois phonétiques et analogie. La régularité vient de ce que "les différentes unités qui comportent un phonème sont régulièrement frappées " (REICHLER-BEGUELIN, 1980, p. 25), affirme Saussure dans ce cours de phonétique. Ces différentes unités sont les " mots » ou les " morphèmes ", et c'est pourquoi, précisément, la régularité est à certains égards, selon le terme du deuxième cours, une « illusion ": l'unité du changement phonétique n'est pas le mot, mais le son; autrement dit, la régularité n'apparaît comme telle que du point de vue de l'idiome, mais linguistiquement, il faut concevoir les choses en termes d'unité distincte de l'unité synchronique constitutive de la langue comme système de signes : l'unité diachronique, ou phonétique (ni phonologique, car l'unité phonologique, selon Saussure, est panchronique, ni significative, car l'unité significative est exclusivement synchronique). On lit notamment dans le deuxième cours, à propos des lois phonétiques:

On est amené à se demander si tous les mots placés dans les mêmes conditions les subissent : sont<-elles> absolues, sans exception ? C'est là qu'on a vu le nœud de la question ; mais il n'est pas là, il est dans la question des unités : faut-il les concevoir comme s'appliquant à telles <ou telles>unités ou non ? $<$ Il n'y a pas de loi si on ne peut indiquer une quantité de faits individuels qui s'y rattachent $;><$ mais $>$ si on va au fond de la loi phonétique il n'est pas dit qu'on ait à envisager la chose ainsi. On dit : tous les mots sont frappés ; on commence par faire une armée de mots, <on suppose> que les mots sont des individus <tout faits $>$ et on dit <qu'>ils sont frappés par la loi. Mais estce bien les mots qui sont ces unités du phénomène phonétique? <Supposons qu'une corde d'une harpe soit faussée ; il est clair que toutes les fois que dans un morceau on joue de cette corde, il se produit une faute. Mais peut-on dire que par exemple les ré de ce morceau sont faux d'après une loi ? C’est absurde ! À l'octave ce ne sera déjà plus vrai !> Nous pouvons nous faire une idée très sensible des lois phonétiques < autrement que sur le papier.> Dans une région, on faussera l'a : on dira se fôcher pour se fâcher. Est-ce des mots qui sont frappés ou bien est-ce <un son> comme dans l'exemple de la corde 
On retrouve donc ici, d'une autre manière, la rupture avec la représentation de la langue comme entité, au profit d'une construction de différents objets - ici, l'unité diachronique par opposition à l'unité synchronique.

Saussure reprend à son compte les qualificatifs d'aveugle et de régulier, mais pour leur conférer une signification nouvelle : aveugle est une conséquence du concept de valeur, dans le cadre duquel le signe ne saurait être que synchronique (" morphologique " implique "synchronique ", et réciproquement ; de même, "phonétique " implique " diachronique ", et réciproquement) ; régulier renvoie à l'effet, du point de vue de l'idiome, de la nature de l'unité diachronique, qui est donc une unité phonétique, ce que Saussure appelle dans le deuxième cours (SAUSSURE, 1997, p. 36) les "unités irréductibles ". À ces deux qualificatifs correspondent, respectivement, une redéfinition du changement comme fonctionnement, et une inscription du changement dans le fonctionnement.

La redéfinition du changement comme fonctionnement est notamment lisible dans la première citation produite dans cet article, extraite de "De l'essence double du langage ", et où Saussure parlait de " [1]'échange, comme seule expression véritable de tout mouvement dans la langue "(SAUSSURE, 2002, p. 60). Le changement, qu'il soit phonétique ou "significatif ", apparaît en effet, dans le cadre de la théorie saussurienne de la langue, comme un phénomène synchronique. La langue, définie comme fonctionnement, ne consiste en rien d'autre qu'en l'articulation (la délimitation-combinaison) à laquelle se résume l'existence de chaque signe (au sens large : c'est-à-dire incluant aussi bien les faits de syntaxe, etc., autrement dit au sens de "fait signifiant ", quel que soit le fait en question) à chaque instant. Or, cette articulation est aussi bien, à chaque instant, un " échange ", produisant, à force, des « changements ».

Concernant l'inscription du changement dans le fonctionnement définitoire de la langue, il faut signaler que la théorisation saussurienne du changement linguistique est très limitée, comme Saussure le reconnaissait lui-même. Rompant avec la représentation de la langue comme entité, Saussure mettait cependant en place un cadre de théorisation, dans le cadre duquel la réflexion sur le changement linguistique gagnerait à être inscrite, pour ne pas mobiliser des entités imaginaires. La théorisation structuraliste du changement linguistique a cependant réintroduit le cadre organiciste des 
premiers comparatistes, comme nous allons le voir dans ce qui suit.

\title{
Loi phonétique et structuralisme
}

Bien que relativement différentes les unes des autres, les élaborations jakobsonienne, martinettienne et hjelmslevienne s'accordent à récuser la conception d'une évolution phonétique " aveugle ", au profit d'une explication structurale du changement. Jakobson affirme ainsi par exemple dans Six leçons sur le son et le sens (1942-1943 ; JAKOBSON, 1976):

\begin{abstract}
Mais nous ne suivons pas Saussure dans son idée préconçue que l'évolution phonique n'a rien à faire avec les valeurs linguistiques des sons. Saussure attribuait aux changements des sons un caractère aveugle, fortuit et "étranger au système de la langue ». L'expérience nous montre au contraire que les changements ne peuvent être compris qu'en fonction du système phonologique qui les subit. Par conséquent, le système des sons en tant que valeurs linguistiques peut être étudié dans son évolution aussi bien que dans son état donné, et la phonologie englobe l'étude historique des phonèmes (JAKOBSON, 1976, p. 62-63).
\end{abstract}

À cet égard, et significativement, Jakobson associe Saussure aux Néogrammairiens, dont la conception des lois phonétiques lui paraît mécanistique, mécanicisme auquel il entend pour sa part substituer une approche téléologique. On lit ainsi dans «Quelles sont les méthodes les mieux appropriées à un exposé complet et pratique de la phonologie d'une langue quelconque ?" (1927, JAKOBSON, 1971), texte signé également par Karcevskij et Troubetzkoy:

Ce n'est pas en renonçant à la notion de « loi phonétique » qu'on dépasserait la tradition des " Junggrammatiker ", mais bien en interprétant cette notion téléologiquement et en abandonnant la conception mécanistique. C’est, en particulier, dans la mesure où les changements phonétiques ont été traités sans considération du système phonologique qui les subit qu'on n'est pas arrivé à atteindre les lois de la phonétique générale (JAKOBSON, 1971, p. 6).

Aveugle est alors notamment associé à "fortuit ", mais également à " destructeur ». Cette deuxième association est au centre de l'élaboration et de la critique martinettiennes. Pour Martinet, en effet, aveugle signifie en particulier « destructeur ». Il écrit par exemple dans Évolution des langues et 
reconstruction (1973-1975; MARTINET, 1975):

On sait que Ferdinand de Saussure, dans son désir de fonder une linguistique générale autonome, avait opposé, avec une vigueur et une insistance remarquables, synchronie à diachronie et marqué le caractère prioritaire de l'une par rapport à l'autre. À une époque où l'on concevait les changements - et l'on pensait " changements phonétiques " - comme se réalisant aux dépens de l'intégrité de la langue, une structure linguistique ne se concevait qu'en synchronie, puisque l'évolution proprement dite ne pouvait qu'être destructrice de cette structure. C'est bien là le point de vue qui est explicité dans le Cours de linguistique générale (MARTINET, 1975, p. 6-7).

On retrouve, dans ce cadre, la notion de changement explicable par le système, sous la forme d'un postulat fonctionnaliste - les formulations plus proprement structurales ne sont d'ailleurs pas absentes des textes de Martinet. Martinet poursuit ainsi:

\begin{abstract}
Pour dépasser ce point de vue, il a fallu, avec la phonologie, intégrer à la structure linguistique la substance phonique pertinente, c'est-à-dire ne plus la concevoir comme une masse amorphe exposée à toutes les pressions du monde physique, mais comme susceptible de réagir à ces pressions en les laissant triompher là où elles pouvaient le faire sans danger pour la communication, mais en s'opposant à elles là où elles auraient pu affecter le fonctionnement de la langue. Ailleurs, elle en retardera les effets assez longtemps pour que l'évolution de la langue sur d'autres points rende le changement, non seulement inoffensif, mais bénéfique. En effet, il représentera alors une économie d'énergie pour l'usager qui n'aura plus désormais à résister à une pression, pour satisfaire ses besoins de communication. Que les changements linguistiques se produisent sur un plan quelconque, lexical, syntaxique, morphologique ou phonologique, de la structure, ils sont toujours, sinon totalement déterminés, du moins toujours contrôlés par la nécessité, pour la langue, d'assurer la communication entre ceux qui la pratiquent. Il n'y a donc aucune incompatibilité entre structure et évolution (MARTINET, 1975, p. 7).
\end{abstract}

Dans cette perspective, la régularité des changements phonétiques paraît à Martinet une conséquence de la double articulation, notion qu'il présente en 1964 dans "Les problèmes de la phonétique évolutive " (MARTINET, 1975) comme une "version synchronique " (MARTINET, 1975, p. 51) de cette régularité : la double articulation vient médier le rapport son/sens, protégeant ainsi le signifiant des atteintes du sens.

Hjelmslev, enfin, refuse de même la notion d'une évolution aveugle, au profit de l'explication structurale du changement. Citons par exemple 
"Structure générale des corrélations linguistiques " (1933, HJELMSLEV, 1985):

\begin{abstract}
On a estimé longtemps que le système grammatical d'une langue n'est rien que le résultat fortuit d'une évolution aveugle. On a oublié de se demander comment il faut expliquer le fait incontestable que, bien que soumise à des altérations constantes, une langue conserve toujours la faculté de former système. On a considéré comme futiles les problèmes des lois générales dirigeant cette force organisatrice de la langue. On a voulu nier - le plus souvent implicitement - l'existence même de telles lois. Ce préjugé a empêché une attitude empirique (HJELMSLEV, 1985, p. 31).
\end{abstract}

C'est pourquoi - et c'est par ailleurs une des singularités de son élaboration -, il redéfinit les " lois phonétiques " (diachroniques) comme des « lois synchroniques", substituant, autant que faire se peut, l'abstraction au temps, réélaboration qui aboutit à une définition formelle de la parenté linguistique, en termes de "fonctions des éléments ", exposée dans Sproget (Le langage, HJELMSLEV, 1966).

Il apparaît ainsi que, quelle que soit l'élaboration, le caractère aveugle des changements linguistiques est réfuté au profit d'une construction structurale et d'une représentation en termes d'entité et de prise du locuteur sur la langue. Plus précisément, cette réfutation n'est possible que dans le cadre d'une telle représentation, qui est celle de la connaissance commune: celle d'une langue conçue comme un instrument de communication, et, comme telle, comme une structure d'appariement du son et du sens et comme une entité dotée d'une existence dans le temps. La représentation en termes d'entité fournit en effet un support à la représentation structurale, qui a valeur explicative en diachronie, et à une réflexion en termes de prise du locuteur sur la langue, dans le cadre de la problématique des rapports son/ sens. Dans la même mesure, cependant, comme nous l'avons montré à une autre occasion (Toutain à paraître), cette représentation est contradictoire, la langue apparaissant tout à la fois comme un instrument de communication, manié par les locuteurs, qui la modifient - fût-ce " inconsciemment»-, et comme une structure en évolution, représentation structurale qui est par ailleurs elle-même problématique, dans la mesure où la structure est toujours constituée à partir d'un état, et où cette constitution implique nécessairement des discontinuités. Comme il apparaît de manière très nette, par exemple, chez Hjelmslev, la continuité est artificiellement rétablie, ou plus exactement, ces discontinuités sont palliées, recouvertes par la représentation organiciste, qui nous reconduit à la linguistique comparatiste, avec laquelle 
les structuralistes entendaient pourtant précisément prendre leurs distances. Citons, pour son caractère tout particulièrement remarquable, l'avantdernier alinéa des conférences de 1934 sur le changement linguistique (HJELMSLEV, 2016):

\begin{abstract}
Mais le langage vit et cela me parait être une découverte nouvelle. Personne ne pourra dire qu'en isolant le système et en le considérant pour lui-même, faisant provisoirement abstraction des autres domaines du langage nous avons mis de côté la vie du langage. C'est le contraire qui s'est produit. Nous avons posé le doigt sur le pouls du langage. Les Anciens n'avaient pas tort de soutenir que le langage est un organisme menant sa vie intérieure autonome (HJELMSLEV, 2016, p. 181-182).
\end{abstract}

Nous voudrions insister, pour conclure, sur cette double contradiction : celle d'un retour paradoxal à la conception néogrammairienne, en dépit d'une volonté de rupture avec celle-ci, et celle de la représentation ellemême, que nous venons de signaler très brièvement. Ces deux contradictions n'en font en réalité qu'une : le concept saussurien de loi phonétique n'est pas purement et simplement un emprunt aux Néogrammairiens, mais il est le creuset de l'élaboration de la distinction synchronie/diachronie, instrument d'une rupture avec la représentation en termes d'entité et, corrélativement, de la théorisation du rapport son/sens; inversement, les contradictions de l'élaboration structuraliste, dont la critique de ce concept - mal ou non compris - n'est que l'effet de l'adoption d'une problématique pré-saussurienne, témoignent de l'inadéquation de la représentation de la connaissance commune et, par ailleurs, de la puissance de l'obstacle épistémologique de l'idiome.

\title{
Références
}

DECIMO, Marc. Saussure à Paris. Cahiers Ferdinand de Saussure, Genève, n. 48, p. 75-90, 1994.

HJELMSLEV, Louis. [1966]. Le langage. Paris: Éditions de Minuit, 1984.

Nouveaux essais. Paris: Puf, 1985.

Système linguistique et changement linguistique. Paris:

Classiques Garnier, 2016. 
JAKOBSON, Roman. Selected Writings $\mathbf{I}^{1}$. La Haye, Paris: Mouton, 1971.

. Six leçons sur le son et le sens. Paris: Éditions de Minuit, [1976] 1991.

MARTINET, André. Évolution des langues et reconstruction. Paris: Puf, 1975.

REICHLER-BEGUELIN, Marie-José. Le consonantisme grec et latin selon F. de Saussure: le cours de phonétique professé en 1909-1910. Cahiers Ferdinand de Saussure, Genève, n. 34, p. 17-97, 1980.

SAUSSURE, Ferdinand de. Deuxième cours de linguistique générale, d'après les cahiers d'Albert Riedlinger et Charles Patois. Oxford; New York; Seoul; Tokyo: Pergamon, 1997.

Écrits de linguistique générale. Paris: Gallimard, $2002^{2}$.

Ferdinand de Saussure: Notes préparatoires pour le cours de linguistique générale 1910-1911, Émile Constantin: Linguistique générale. Cours de M. le professeur de Saussure 1910-1911. Cahiers Ferdinand de Saussure, Genève, n. 58, p. 83-289, 2005.

TOUTAIN, Anne-Gaëlle. À paraître. Structuralisme et organicisme: une analyse épistémologique. Cahiers de l'ILSL.

DOI - http://dx.doi.org/10.5902/2179219436582

1 Il s'agit de l'édition augmentée d'addenda. La première édition des Selected Writings I date de 1962 .

2 Signalons que toutes nos citations de cet ouvrage ont été corrigées sur les manuscrits. Le texte peut donc différer de celui de ce dernier. 